\title{
Zur Theorie der Rotationsspektren ${ }^{1}$ ).
}

\section{Von Fritz Reiche.}

Mit einer Abbildung.

(Eingegangen am 29. Dezember 1919.)

Bekanntlich schreibt man die im langwelligen Ultrarot gelegenen Absorptions- und Emissionsbanden mehratomiger Gasverbindungen der Rotation ihrer Moleküle zu, deren Atome polare elektrische Ladungen tragen und daher bei der Rotation des Moleküls nach der klassischen ElektrodynamikStrahlung emittieren und absorbieren. Seit N.Bjer rum ${ }^{2}$ ) weiß man, daß diese Rotationen sich auch im kurzwelligen Ultrarot bemerkbar machen. Existiert nämlich eine in dieses Gebiet fallende Schwingung der Atome im Molekül gegeneinander, etwa mit der Schwingungszahl $\nu_{0}$, und rotiert anßerdem das ganze Molekül mit der Umlaufszahl $\nu_{r}$. (pro Sekunde), so entstehen durch die Zusammensetzung der Schwingung mit der Rotation nach Rayleigh ${ }^{3}$ ) neben den beiden alten Absorptions- (und Emissions-) stellen $\nu=\nu_{0}$ und $\nu=\nu_{r}$ zwei nene: $\nu=\nu_{0}+\nu_{r}$ und $\nu=\nu_{0}-\nu_{r}$. In diesem Sinne hat bekanntlich Bjerrum die oft beobachtete „Doppelbande" gedeutet.

Nach der Quantentheorie, und zwar in der ersten Planckschen Fassung, auf die wir uns hier beschränken wollen ${ }^{4}$ ), kann jedoch das Molekül nur mit ganz bestimmten, diskreten Umlaufszahlen $\nu_{r}^{(n)}$ rotieren. Die entsprechenden diskreten Werte seiner Rotationsenergie seien $E_{r}^{(n)}$. Daher treten dann - so wurde gefolgert - in seinem $\mathrm{Ab}$ sorptions- und Emissionsspektrum die folgenden "Linien" auf: erstens im langwelligen Ultrarot. eine Serie $\nu=v_{r}^{(n)}(n=0,1,2,3, \ldots)$ und zweitens im kurzwelligen Ultrarot die beiden Serien: $\nu=\nu_{0}+\nu_{r}^{(n)}$ $(n=0,1,2,3, \ldots)$ und $\nu=\nu_{0}-v_{r}^{(n)}(n=0,1,2,3, \ldots)$. Fïr das

1) Die vorliegende Arbeit war im wesentlichen fertig gestellt, als eine $\mathbf{A b}$ handlung von W. Lenz (Verh. d. D. Phys. Ges. 21, 632, 1919) erschien, in der die Theorie der Bandenspektren allgemein auf Grund der auch hier befolgten, auf K. Schwarzschild (Sitzungsber. d. Berl. Akad. d. Wiss. 1916, S. 548) zurückgehenden Methode entwickelt wird. Dort wird auch erwähnt, daß die Theorie der ultraroten Rotationsspektren sich der allgemeinen Theorie einfügt. Vgl. ferner eine inzwischen erschienene Abhandlung von T. Heurlinger, Zs. f. Phys. 1, 82, 1920.

2) N. Bjerrum, Nernst-Festschrift, Halle 1912, 8. 90.

3) Lord Rayleigh, Phil. Mag. (5) 24, 410, 1892.

4) Vom Standpunkt der zweiten Planckschen Theorie (klassisehe Absorption) hat Planck selbst das Problem behandelt (Ann. d. Phys. 52, 491, 1917; 53, 241, 1917). 
einfachste zweiatomige Molekülmodell (starre „Hantel“; Drehung um eine zur Verbindungslinie der Atome senkrechte feste Achse) liefert die Quantentheorie ${ }^{1}$ ):

$$
\left.\begin{array}{l}
v_{r}^{(n)}=\frac{n h}{4 \pi^{2} J} \\
E_{r}^{(n)}=\frac{n^{2} h^{2}}{8 \pi^{2} J}
\end{array}\right\}(n=0,1,2,3, \ldots)
$$

wo $h$ die Plancksche Konstante und $J$ das Trägheitsmoment des Moleküls ist. Hiernach ist

$$
E_{r}^{(n)}=n \frac{h \nu_{r}^{(n)}}{2} \text { (Planck-Ehrenfestscher Ansatz) }
$$

während Bjerrum auf Gruud des etwas abgeänderten (H. A. Lorentzsehen) Ansatzes

die Werte:

$$
E_{r}^{(n)}=n . h v_{r}^{(n)}
$$

$$
\left.\begin{array}{l}
\nu_{r}^{(n)}=\frac{n h}{2 \pi^{2} J} \\
E_{r}^{(n)}=\frac{n^{2} h^{2}}{2 \pi^{2} J}
\end{array}\right\}
$$

benutzt ${ }^{2}$ ). In jedem Falle bilden die $v_{r}^{(n)}$ eine Reihe äquidistanter Schwingungszahlen, mit dem Abstand $\frac{h}{4 \pi^{2} J}\left(\right.$ bzw. $\left.\frac{h}{2 \pi^{2} J}\right)$, and dieselbe Aquidistanz findet sich in den beiden Serien $\nu=\nu_{0} \pm \nu_{r}^{(n)}$ wieder.

Die Experimentaluntersuchungen von Eva v. Bahrs ${ }^{3}$ am Chlorwasserstoff und Wasserdampf, die von A. Eucken ${ }^{4}$ ) im obigen Sinne ausführlich diskutiert wurden, und die eingehenden Messungen vou H. Rubens and G. Hettner ${ }^{5}$ ) am Wasserdampf haben die Existenz und Aquidistanz der prophezeiten Linien erwiesen. Bei dieser Auffassung werden also die im langwelligen Ultrarot gelegenen Linien direkt mit den diskreten, quantenmäBig ausgewählten Umlaufszablen $\nu_{r}^{(n)}$ identifiziert.

Schon K. Schwarzschild ${ }^{6}$ ) zeigte, daß dieses Vorgehen zu „klassisch" ist, und daß es vom Standpunkt der Quantentbeorie und

1) Vgl. z. B. M. Planck, Ann. d. Phys. 50, 390, 1916.

2) N. Bjerrum, Verh. d. D. Phys. Ges. 16, 640, 1914.

3) E. v. Bahr, Ebenda 15, 731, 1150, 1913.

4) A. Eucken, Ebenda 15, 1159, 1913.

5) H. Rubens und G. Hettner, Sitzungsber. d. Berl. Akad. d. Wiss. 1916, S. 167

6) K. Schwarzschild, Sitzungsber. d. Berl. Akad. d. Wiss. 1916, \$. 548. 
im Sinne der Bohrschen Auffassung von der Linienemission konsequenter ist, wenn man folgende Annahme macht: das Molekül gehe aus einem durch die Quantenzahl $m$ definierten Quantenzustand mit der Rotationsenergie $\boldsymbol{E}_{r}^{(m)}$ in einen anderen Quantenzustand über, der durch die Quantenzahl $n$ charakterisiert ist, und dem die Energie $E_{r}^{(n)}<E_{r}^{(m)}$ entspricht. Dann wird, nach der „Bohrschen Frequenzbedingung", eine monochromatische Strahlung von der Schwingungszahl

$$
\nu_{r}=\frac{E_{r}^{(m)}-E_{r}^{(n)}}{h}
$$

emittiert. Bei dem umgekehrten Ubergang wird Strahlung von dieser Sehwingungszabl absorbiert. Man kann hier für $E_{r}^{(n)}$ die Formel (1) oder (1 a) einsetzen. Indessen wollen wir das Molekülmodell noch etwas verallgemeinern: wir betracbten das Molekül als einen symmetrischen Kreisel mit den Trägheitsmomenten $J, K=J, L$. Um der Schwierigkeit eines „entarteten" Problems zu entgehen, nehmen wir an, daß die Bewegung des Kreisels in einem schwachen "Richt-

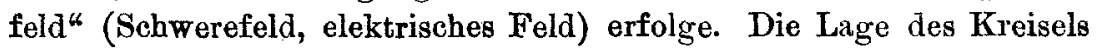
sei durch die drei Eulerschen Winkel $\vartheta$ (Neigungswinkel der Figurenachse), $\psi$ (Präzessionswinkel) und $\varphi$ (Eigendrehungswinkel) definiert, denen die drei Quantenzahlen $n_{1}(\geqq 0), n_{2}(\geqq 0)$ und $n_{3}(\geqq 0)$ entsprechen. Es ist also $\frac{n_{3} h}{2 \pi}$ der Drehimpuls des Kreisels um seine Figurenachse; zn dieser Achse gehört das Trägheitsmoment $L$. Dann gelten für die quantenmäßig ausgezeichneten Energiewerte, bei verschwindend kleinem Richtfeld, folgende Ausdrücke $\left.{ }^{1}\right)$ :

$$
\left.\begin{array}{l}
E_{r}^{\left(n_{1} n_{2} n_{3}\right)}=\frac{h^{2}}{8 \pi^{2}}\left\{\frac{\left(n_{1}+n_{2}\right)^{2}-n_{3}^{2}}{J}+\frac{n_{3}^{2}}{L}\right\} \text { für } n_{2} \geqq n_{3} \\
E_{r}^{\left(n_{3} n_{2} n_{3}\right)}=\frac{h^{3}}{8 \pi^{2}}\left\{\frac{\left(n_{1}+n_{3}\right)^{2}-n_{3}^{2}}{J}+\frac{n_{3}^{2}}{L}\right\} \text { für } n_{2}<n_{3}
\end{array}\right\}
$$

Die beim Ubergang aus dem Quantenzustand $\left(m_{1} m_{2} m_{3}\right)$ in den Quantenzustand $\left(n_{1} n_{2} n_{\mathrm{B}}\right)$ emittierte, oder beim umgekehrten Utbergang absorbierte Strahlung hat dann die Schwingnngszahl:

$$
\nu_{r}=\frac{E_{r}^{\left(m_{1} m_{2} m_{3}\right)}-E_{r}^{\left(n_{1} n_{2} n_{3}\right)}}{h}
$$

Wir betrachten im folgenden nur zweiatomige Moleküle (wie $\mathrm{HCl}, \mathrm{HBr}, \mathrm{CO}$ ). Bei ihnen ist stets das Trägheitsmoment $L$ um die Figurenachse sehr klein gegen die beiden anderen, also

$$
J \geqslant L
$$

1) Vgl. F. Reiche, Ann. d. Phys. 58, 668, 1919. 
Benatzen wir die Abkürzangen:

so ist also:

$$
\alpha=\frac{h}{8 \pi^{2} J}, \quad \beta=\frac{h}{8 \pi^{2} L}
$$

$$
\beta \gg \alpha
$$

Berücksichtigt man diese Ungleichung, so erkennt man sofort, daß für alle hier in Frage kommenden Ubergänge des Moleküls aus dem einen in den anderen Quantenzustand stets

$$
m_{3}=n_{3}
$$

sein muß. Wäre diese Gleichung nämlich nicht erfüllt, so würde nach (5) und (4) in $\nu$ ein Glied $\beta\left(m_{3}^{2}-n_{3}^{2}\right)$ auftreten, das infolge der Ungleichung (8) für die Größenordnung von $\nu$ allein ausschlaggebend ist; es würden sich dann für $v$ Werte ergeben, die ins sichtbare Gebiet fallen. Aus (4), (5), (7) und (9) folgt, wenn wir noch abkürzend:

$$
\frac{\nu_{r}}{\alpha}=x_{r}
$$

setzen:

$$
\begin{aligned}
& x_{r}=\left(m_{1}+m_{2}\right)^{2}-\left(n_{1}+n_{2}\right)^{2} \text { für }\left\{\begin{array}{c}
m_{2} \geqq m_{3} \\
n_{2} \geqq n_{3}
\end{array}\right. \\
& x_{r}=\left(m_{1}+m_{3}\right)^{2}-\left(n_{1}+n_{3}\right)^{2} \text { für }\left\{\begin{array}{c}
m_{2}<m_{3} \\
n_{2}<n_{3}
\end{array}\right. \\
& x_{r}=\left(m_{1}+m_{2}\right)^{2}-\left(n_{1}+n_{3}\right)^{2} \text { für }\left\{\begin{array}{c}
m_{2} \geqq m_{3} \\
n_{2}<n_{3}
\end{array}\right. \\
& x_{r}=\left(m_{1}+m_{3}\right)^{2}-\left(n_{1}+n_{2}\right)^{2} \text { für }\left\{\begin{array}{c}
m_{2}<m_{3} \\
n_{2} \geqq n_{3}
\end{array}\right.
\end{aligned}
$$

Dafür kann man, da $m_{1}$ und $n_{1} \geqq 0$ sind, zusammenfassend schreiben:

$$
\left.x_{r}=p^{2}-q^{2}, \quad \text { wo } \quad \begin{array}{c}
p \\
q
\end{array}\right\} \geqq m_{\mathrm{g}} .
$$

Es fragt sich non, welche Zahlenkombinationen von $p$ und $q$, d. h. welche Ubergänge nach den quantentheoretischen „Auswahlprinzipien" möglich sind. Nach dem Bohrschen Analogieprinzip I) muß für hohe Quantenzahlen $p$ und $q$ Anschln $B$ an die klassische Theorie gewonnen werden. Setzt man also

so folgt:

$$
\begin{gathered}
p-q=s, \quad \text { wo } \quad s \leqslant q, \\
x_{r}=2 q s+s^{2} \sim 2 q s .
\end{gathered}
$$

1) N. Bohr, On the quantum theory of line-spectra. D. kgl. Dansk. Vidensk. Selsk. Bkrifter. Naturvidensk. og Mathem. Afd. 8, Raekke IV, 1. Kopenhagen 1918. 
Nach der klassischen Theorie aber muß die emittierte oder absorbierte Linie mit der Umlaufszahl1) übereinstimmen, also bei $\nu_{.}=\nu_{r}^{(q)}$ liegen. Nun ist aber hier nach (1):

also

$$
v_{r}^{(q)}=\frac{q h}{4 \pi^{2} J}=2 \alpha q
$$

$$
x_{r}=\frac{v_{r}^{(q)}}{\alpha}=2 q \text {. }
$$

Soll dies mit (14) übereinstimmen, so muß $s=1$ sein. Es sind also nur solche Übergänge möglich, für die

$$
p-q=1
$$

ist. Zu demselben Ergebnis führt auch das von A. Rubinowicz ${ }^{2}$ ) ausgearbeitete Auswahlprinzip, das sich auf den Satz von der Erhaltung des Drehimpulses beim Emissions- und Absorptionsakt gründet. Hieraus und aus (12) folgt:

$$
x_{r}=2 q+1, \text { wo } q \geqq m_{3} .
$$

Aus dieser Formel folgt unter Beachtung von (10), daß die von der Rotation allein herrührenden Absorptions- und Emissionsstellen in der Tat Reihen diskreter äquidistanter Linien darstellen.

Die im kurzwelligen Ultrarot gelegenen Absorptionsstellen sind nach der klassischen Theorie, wie schon oben gesagt, die folgenden:

und

$$
\left.\begin{array}{l}
\nu=v_{0}+\nu_{r}^{(n)}=\nu_{0}+n \frac{h}{4 \pi^{2} J} \\
\nu=\nu_{0}-v_{r}^{(n)}=\nu_{0}-n \frac{h}{4 \pi^{2} J}
\end{array}\right\}(n=0,1,2,3, \ldots)
$$

wo $\nu_{0}$ die Schwingungszahl der Atomkerne im Molekül darstellt. Dieses von der klassisehen Theorie geforderte "Kombinationsgesetz" bleibt auch bei der quantentheoretischen Betrachtung erhalten, wenn man die in den praktischen Fällen zutreffende Voraussetzung macht, $\mathrm{da} B$ die Schwingungsamplitude jedes der beiden Atomkerne klein ist gegen den mittleren Abstand des betreffenden Atomkernes vom Schwerpunkt des Moleküls. Dann setzt sich nämlich, wie man zeigen kann, die Gesamtenergie des Moleküls in erster Näherung additiv aus der

1) Wenn $m_{3}\left(=n_{3}\right)$ von Null verschieden ist, so führt das Molekül keine Rotation wie im Falle $m_{3}=n_{3}=0$ aus, sondern eine reguläre Präzession. An die Stelle der Umlaufszah] tritt dann die Größe: $\frac{1}{2 \pi}$. Präzessionsgesohwindigkeit, die, wie leicht zu sehen, ebenfalls den Wert 2 a $q$ besitzt.

2) A. Rubinowicz, Phys. ZS. 19, 441, 465, 1918. 
Atomschwingungsenergie und der Rotationsenergie zusammen, in Zeichen :

$$
E=E_{s}+E_{r}
$$

wo $E$ die gesamte, $E_{s}$ die Schwingungsenergie darstellt. Für die Energie $E_{s}$ der linearen monochromatischen Schwingung $\left(\nu_{0}\right)$ setzen wir im Sinne der ersten Planckschen Theorie den Quantenwert:

$$
E_{s}=n_{4} h \nu_{0}\left(n_{4}=0,1,2,3,4, \ldots\right)
$$

für $E_{r}$ den dưch (4) gegebenen Quantenwert. Dann ist die Gesamtenergie $E$ durch vier Quantenzahlen $n_{1}, n_{2}, n_{3}, n_{4}$ bestimmt, and die Bohrsche Frequenzbedingung liefert als emittierte oder (beim umgekehrten Ubergang) absorbierte Schwingungszahl den Ausdruck:

$$
\nu=\frac{E^{\left(m_{1} m_{2} m_{3} m_{4}\right)}-E^{\left(n_{1} n_{2} n_{3} n_{4}\right)}}{h} .
$$

Unter Beachtung der vorangehenden Entwickelungen, speziell mit Benutzung von (9), (10), (12), folgt dann:

wo

$$
\left.\begin{array}{l}
\nu=\left(m_{4}-n_{4}\right) \nu_{0}+z \cdot \alpha, \\
\left.z=p^{2}-q^{2} \text { und } \begin{array}{l}
p \\
q
\end{array}\right\} \geqq m_{3}
\end{array}\right\}
$$

Für die rein harmonische monochromatische Schwingung $\left(\nu_{0}\right)$ folgt nun aber ans den von Rabinowicz und Bohr auggearbeiteten Auswahlprinzipien, daß ibre Quantenzahl $n_{\mathbf{q}}$ bei Quantenübergängen sich nur um $0,+1,-1$ ändert. Also kann $m_{4}-n_{4}$ nur die Werte $0,+1,-1$ annehmen. Der erste Fall $\left(m_{4}=n_{4}\right)$ führt auf die schon betrachteten Emissions- und Absorptionsstellen zarück. Physikalisch Neues dagegen liefert der Fall: $n_{4}-n_{4}=1$, nämlich die Schwingungszahlen:

wo

$$
\left.\begin{array}{l}
\nu=\nu_{0}+\varepsilon \cdot \alpha, \\
\left.\varepsilon=p^{2}-q^{2} \text { und } \begin{array}{c}
p \\
q
\end{array}\right\} \geqq m_{3}
\end{array}\right\}
$$

Das Bohrsehe Analogieprinzip fordert, daß für hohe Quantenzahlen Anschluß an die klassische Theorie gewonnen wird, daß also für

$$
p-q=s \leqslant q
$$

nur die Absorptions- und Emissionstellen: $\nu=\nu_{0}+v_{r}^{(q)} ; \nu=\nu_{0}-v_{r}^{(q)}$ resultieren ${ }^{1}$ ). Da nun (22) in diesem Falle die Gestalt:

$$
\nu \sim \nu_{0}+2 \alpha q s=\nu_{0}+\nu_{r}^{(q)} . s
$$

1) Die Mittellinie $\nu=\nu_{0}$ verschwindet, wenn - was wir in folgenden voraussetzen - der rotationslose Zustand nichtexistiert. 
annimmt, so folgt: $s$ kann nur die Werte $+1,-1$ besitzen. Also wird allgemein, nach (22):

$\left.\begin{array}{lll}\text { für } p=q+1: & v=\nu_{0}+\alpha(2 q+1), \quad \text { wobei } & q \geqq m_{3} \\ \text { für } p=q-1: & v=\nu_{0}+\alpha(-2 q+1), \quad, \quad q \geqq m_{3}+1\end{array}\right\}$

was man unter Berücksichtigung von (7) zusammenfassend so schreiben kann:

$$
\nu=\nu_{0} \pm\left(q+\frac{1}{2}\right) \frac{h}{4 \pi^{2} J}
$$

Wo

$$
q \geqq m_{3} \text {. }
$$

Die entsprechenden Linienbilder sind in der Figur gezeichnet. Dabei ist die Mitte $\left(\nu=\nu_{0}\right)$ durch 0 gekennzeichnet. Die Linien sind, bis auf die UnregelmäBigkeit in der'Mitte, äquidistant. Ihr Abstand ist:

$$
\Delta \nu=2 \alpha=\frac{h}{4 \pi^{2} J}
$$

also ebenso groß, wie der Abstand zweier aufeinander folgender Umlaufszahlen nach (1). Aber das Lagenbild der Linien ist wesentlich verschieden von dem klassischen Lagenbild (17).

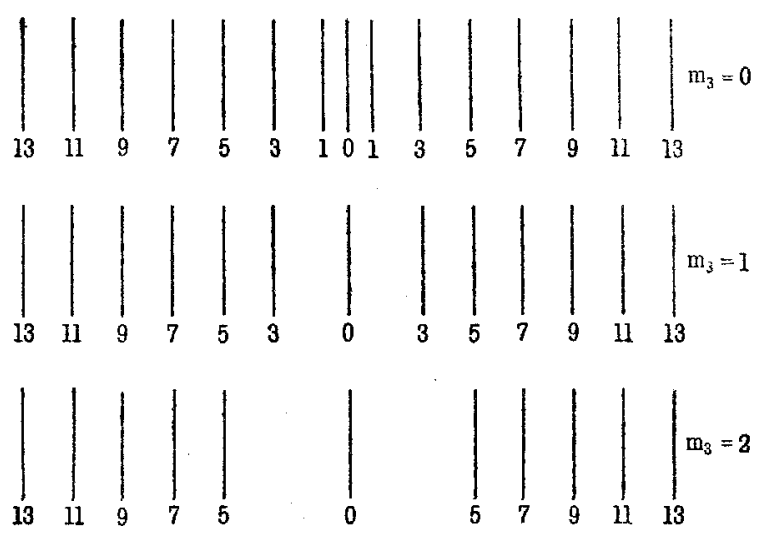

Für die Prüfung dieser Resultate an der Erfahrang kommen leider nur die Messungen Eva v. Bahrs') am Chlorwasserstoff in Betracht, die vermutlich durch Versuchsfehler entstellt sind. Auf den Wasserdampf, für den präzisere Messungen (E. v. Bahr, H. Rubens und G. Hettner) vorliegen, kann man wegen der Dreiatomigkeit seines Moleküls die vorliegende Theorie nicht ohne weiteres anwenden.

I) E. v. Bahr, Verh. d. D. Phys. Ges. 15, $1150,1913$. 
Vergleicht man das Lagenbild der E. v. Bahrschen Linien, soweit dies bei der Ungenauigkeit der Messungsergebnisse möglich ist, mit den theoretischen Lagenbildern in der Figur, so geht eins wohl mit Deutlichkeit daraus hervor: die Fälle $m_{3}=n_{3}=2$ und diejenigen, die noch höheren Werten von $m_{3}=n_{3}$ entsprechen, kommen wegen der großen und mit wabhenden $m_{3}$-Werten sich vergrößernden Lücke in der Mitte nicht in Betracht. Damit scheinen mir alle diejenigen Modelle des HCl-Moleküls ansgeschaltet, die um die Verbindungslinie der beiden Kerne (H und_Cl) einen rotierenden mehrelektronigen "Bindungsring", also einen mehrquantigen Drehimpuls um die Figurenachse besitzen sollen, wie etwa das Kosselsche ${ }^{1}$ ) HCl-Modell mit seinem Achter-Ring. Dagegen ist es schwer, auf Grund der E. v. Bahrschen Zahlen zwischen den beiden ersten Lagenbildern; $d$. $h$, also zwischen den Fällen $m_{3}=n_{3}=0$ und $m_{3}=n_{3}=1$ die Wahl zu treffen.

Diese Sachlage wird jedoch, wie mir scheint, noch etwas verändert, wenn man die Quantentheorie der Rotationswärmen zweiatomiger Gase zu Hilfe ruft, die ja ebenfalls mit dem Energieausdruck (4) arbeitet 2). Aus ihr ergibt sich folgendes Resultat.

1. Ist $m_{3}=n_{3}=0$, so erhält man für die Rotationswärme (in ihrer Abhängigkeit von der Temperatur) nur dann brauchbare Kurven, wenn die Quantenzahl $q=n_{1}+n_{2}$ den Wert 0 nicht annimmt. Dasselbe gilt natürlich für $p=m_{1}+m_{2}$. Mit einiger Sicherheit kann man auch sagen, daß die Wahrscheinlichkeit $w_{q}$ eines darch die Quantenzahl $q$ charakterisierten Rotationszustandes den Wert besitzt ${ }^{3}$ ):

$$
w_{q}=\text { const } q \cdot e^{-q^{2} \sigma}, \text { wo } \sigma=\frac{h^{2}}{8 \pi^{2} J k T} .
$$

Dabei ist $k$ die Boltzmannsohe Konstante, $T$ die absolute Temperatur. Für $q=0$ ist also $w_{q}=0$. Diese in (26) dargestellte Verteilungsfunktion, die mau erhält, wenn $\operatorname{man} n_{2}=0$,verbietet", liefert, soweit ich sehe, die beste Kurve für die Rotationswärme des Wasserstoffes 4). Aus dem eben Gesagten und aus (23) ergibt sich daher der Schluß, daB die erste Linie links und rechts

1) W. Kossel, Ann. d. Phys. 49, 358 ff., 1916.

2) Vgl. z. B. F. Reiche, Ann. d. Plys. b8, 657, 1919.

3) F. Reiche, 1. c., $\S 9$. Dieselbe Verteilungsfunktion erhielt auch Frau 8. Rotgzayn (Ann.d. Phys. ă7, 81, 1918) auf Grund der zweiten Planckschen Theorie.

d) Wie man aus der 1. c. gezeichneten Fig. 6 erkennt, fällt allerdings der. bei $T=197^{\circ}$ von Eucken und Scheel-Hense gemessene Wert beträchtlich unter die theoretische Kurve. 
von der Mitte (in der Figur, Fall $m_{3}=0$, mit 1 bezeichnet) fortfällt, und sich also das für $m_{3}=1$ gezeichnete zweite Bild antomatisch herstellt.

2. Ist $m_{3}=n_{3}=1$, so erbält man zwar ohne neue Beschränkung der Quantenzahlen für die Rotationswärme eine mit der Temperatur monoton ansteigende, also prinzipiell brauchbare Kurve. Dagegen zeigt es sich, daß diese Kurve, wenn sie bei tiefen Temperaturen den Beobachtangen angepaßt wird, bei hohen Temperaturen bedeutend za tief verläuft. Will man daher auch hier die unter 1 genannte "beste" Kurve erhalten, also zu der Verteilnngsfunktion (26) gelangen, so mu man auch hier $n_{2}=0$ verbieten. Wie man aus (11) erkennt, erleiden jedoch dadurch $p$ and $q$ keine weitere Einschränkung!

So kommt es, daß die Lagenbilder in Fall 1 und 2 identisch werden. Eine Entscheidung zwisehen den Fällen $m_{3}=n_{3}=0$ und $m_{3}=n_{3}=1 \mathrm{zu}$ treffen, ist also hier nicht möglich! Wichtig jedoch ist das Resultat, daß nur das in der Figur an zweiter Stelle stehende Bild für den Vergleich mit den Beobachtungen in Betracht kommt. Unter dieser Annahme sind in der Tabelle aus den von E. v. Bahr beobachteten Mittelwerten von $\nu_{r}$ die Größen $\frac{\nu_{r}^{(1)}}{3}, \frac{\nu_{r}^{(2)}}{\overline{5}}, \frac{\nu_{r}^{(3)}}{7}$ usw. gebildet, die in der dritten Spalte stehen. Ihr Mittel ist 2,79.1011. Mit diesem Mittelwert sind dann in der vorletzten Spalte die $\nu_{r}$ nach (23) berechnet und mit den gemessenen Werten (letzte Spalte) verglichen. Die Ubereinstimmung ist befriedigend.

\begin{tabular}{c|c|c|c|c}
\hline$q$ & $2 q+1$ & $\frac{\nu_{r} \cdot 10-11 \text { (Mittel) }}{2 q+1}$ & $\begin{array}{c}v_{r} \cdot 10-11 \\
\text { berechnet nach (24) }\end{array}$ & $\begin{array}{c}\nu_{r} \cdot 10-11 \\
\text { beobachtet (Mittel) }\end{array}$ \\
\hline 1 & 3 & 2,48 & 8,37 & 7,45 \\
2 & 5 & 2,79 & 13,95 & 13,95 \\
3 & 7 & 2,88 & 19,53 & 20,15 \\
4 & 9 & 2,91 & 25,11 & 26,20 \\
5 & 11 & 2,91 & 30,69 & 32,0 \\
6 & 13 & 2,83 & 36,27 & 36,8 \\
7 & 15 & 2,72 & 41,85 & 40,8 \\
& & & &
\end{tabular}

Der mittlere Abstand benachbarter Linien ergibt sich zu $\Delta \nu=5,58.10^{11}$.

Hieraus und aus (25) folgt unter Benutzung des Wertes $h=6,545 \cdot 10^{-27}$ :

$$
J=2,97.10^{-40} \text {. }
$$


Für den Abstand $\varrho$ des Wasserstoffkerns vom Chlorkern gilt die Formel:

$$
\varrho=\sqrt{\frac{36,46 \cdot J}{35,46 \cdot M_{H}}}=0,78645 \cdot \sqrt{J} \cdot 10^{12}
$$

wo $M_{H}=1,6624.10^{-24}$ die Masse des Wasserstoffkerns ist. Setzt man für $J$ den Wert aus (28) ein, so findet man:

$$
\varrho=1,355 \cdot 10^{-8} \text {. }
$$

Aus der Verteilungsfunktion (26) kann man noch berechnen, für die wievielte Linie (von der Mitte aus gerechnet) $w_{q}$ ein Maximum wird, d.h. wo etwa die Maxima der Absorptionsdoppelbande liegen. Unter Benutzung der Werte:

$h=6,545 \cdot 10^{-27} ; \quad k=1,3723 \cdot 10^{-16}, \quad J=2,97 \cdot 10^{-40} ; \quad T=291$, wird $\sigma \cdot=0,0457$, und daher nimmt $w_{q}$ für $q=1,2,3,4,5 \ldots$ die Werte an: 0,$955 ; 1,666 ; 1,988 ; 1,924 ; 1,593 ; \ldots$ Die Maxima der kannelierten Doppelbande liegen demnach bei der 3. Linie (links und rechts von der Mitte), in guter Ubereinstimmung mit E. v. Bahrs Beobachtung.

Zusammenfassend kann man meines Erachtens mit einiger Sicherheit sagen, daß das HCl-Molekül (und dasselbe gilt natürlich von allen zweiatomigen, heteropolaren Gasverbindungen) keinen, oder höchstens einen einquantigen Drehimpals um seine Längsachse (Verbindungslinie der Kerne) besitzt. Beides spricht gegen das Bohrsche und Kosselsche Modell mit seinem rotierenden Elektronenring, der dem System einen mehrquantigen Drehimpuls verleiht. Die erste Alternative würde dagegen für ein Modell sprechen, ähnlich (oder gleich) demjenigen, das kürzlich F. Haber ${ }^{1}$ ) im Anschluß an M. Borns "Würfelatome" vorgeschlagen hat.

In jedem Falle wäre es wichtig, zwecks genaner Prüfnng der Theorie, die Rotationsspektren der Halogenwasserstoffe einer erneuten, eingehenden Messung zu unterwerfen.

Berlin, Dezember 1919.

Anmerkung bei der Korrektur. Erst inach Abschluß der Korrektur der vorliegenden Arbeit erhielt der Verfasser Kenntnis von neuen Beobachtungen von E. S. Imes (Astroph. Journ. 50, 251, 1919) an den Halogenwasserstoffen, die E. v. Bahrs Messungen an Genauigkeit weit übertreffen. Nach diesen Beobachtungen ist mit voller Dentlichkeit die Lage der Linien durch Formel (17), und

1) F. Habex, Verh. d. D. Phys. Ges. 21, 750, 1919. 
nicht durch Formel (24), gegeben. Die Mittellinie fehlt. Das Ergebnis der Imessohen Messangen ist daher nicht vereinbar mit dem Lagenbild der Linien, das hier au dem einfachsten schematischen Modell auf Grund der üblichen Rotationsquantelung, der Bohrschen Frequenzbedingung and des Bohrschen Analogieprinzips abgeleitet worden ist. Es ist daher zu vermuten, daß eine oder mebrere der Prämissen dieser Ableitung nicht der Wirklichkeit entsprechen.

Herr Prof. A. Einstein, mit dem ich Gelegenheit hatte, öfters über diese Sachlage za sprechen, wies mich auf eine Möglichkeit hin, durch Abänderung der Rotationsquantelung den Widerspruch mit den Beobachtungen zu beheben. Mit seiner freundlichen Erlaubnis gebe ich dies hier bekannt.?

Wènn man für den Drehimpuls des Moleküls - der Einfachheit halber werde das "ebene" Problem betrachtet - an Stelle von $\frac{n h}{2 \pi}$ die Quantenwerte $\left(n+\frac{1}{2}\right) \frac{h}{2 \pi}$ annimmt, also für die Rotationsenergien die Quantenwerte $\frac{\left(n+\frac{1}{2}\right)^{2} h^{2}}{8 \pi^{2} J}$, so ergibt sich anf dem beschrittenen Wege, unter Anwendung der Bohrsehen Frequenzbedingung: und des Analogieprinzips für die Lage der Linien:

$$
\boldsymbol{v}=\boldsymbol{\nu}_{0} \pm(n+1) \frac{h}{4 \pi^{2} J}(n=0,1,2,3, \ldots)
$$

in Übereinstimmang mit den Imesschen Beobachtungen. 